\title{
Neuronal Correlates of the Perceptual Invariance of Words and Other Sounds in the Supplementary Motor Area of Macaques
}

Jonathan Melchor, ${ }^{1}$ Isaac Morán, ${ }^{1}$ José Vergara,${ }^{2}$ Tonatiuh Figueroa,,${ }^{1}$ Javier Perez-Orive,${ }^{3}$ and Luis Lemus ${ }^{1 *}$

${ }^{1}$ Department of Cognitive Neuroscience, Institute of Cell Physiology, Universidad Nacional Autónoma de México (UNAM). 04510. Mexico City, Mexico.

${ }^{2}$ Department of Neuroscience, Baylor College of Medicine, Houston, Texas.

${ }^{3}$ Luis Guillermo Ibarra Ibarra National Rehabilitation Institute. Mexico City, Mexico.

* Corresponding author

lemus@ifc.unam.mx

Telephone: (+52) 5556225675

KEYWORDS: Perceptual constancy, macaques, acoustic recognition, frontal cortex, psychophysics. 


\section{ABSTRACT}

1 The supplementary motor area (SMA) of the brain is critical for integrating memory and

2 sensory signals into perceptual decisions. For example, in macaques, SMA activity correlates

3 with decisions based on the comparison of sounds. ${ }^{1}$ In humans, functional MRI shows SMA

4 activation during the invariant recognition of words pronounced by different speakers. ${ }^{2}$

5 Nevertheless, the neuronal correlates of perceptual invariance are unknown. Here we show that

6 the SMA of macaques associates novel sounds with behaviors triggered by similar learned

7 categories when recognizing sounds such as words. Notably, the neuronal activity at single and

8 population levels correlates with the monkeys' behaviors (e.g. hits and false alarms). Our

9 results demonstrate that invariant recognitions of complex sounds involve premotor computations in areas other than the temporal and parietal speech areas. Therefore, we propose that perceptual invariance depends on motor predictions and not only sensory representations.

We anticipate that studies on speech will observe sensory-motor transformations of acoustic information into motor skills.

INTRODUCTION

17 We typically learn our first words from the voice of our mother. Notably, after that, we are capable of recognizing the same words spoken by multiple speakers. Similarly, the ability to identify versions of learned sounds (vLS) has been studied in songbirds, ferrets, and mice. ${ }^{3-5}$ Rhesus monkeys and chimpanzees acknowledge vocalizations for foods of different values and

21 from various troop members. ${ }^{6,7}$ Although this ability is vital for communication, the neural

22 basis for the invariant recognition of sounds is not well understood. 
23 Current knowledge of how neuronal activity produces the semantic and invariant perception of

24 vocal sounds is also scant. ${ }^{4,8-10}$ For instance, "voice-sensitive" and "vocalization-sensitive" cortical areas have been identified using neurophysiological and imaging studies in humans and nonhuman primates. ${ }^{11-15}$ Additionally, the invariant perception of ferrets generalizing vowel identities $(/ \mathrm{u} /$ and $/ \epsilon /)$ throughout variations of fundamental frequencies, sound levels, and locations has been correlated with auditory cortex activity. ${ }^{4}$ In zebra finches, reports suggest invariant responses for different categories of conspecific vocalizations. ${ }^{9}$ A recent study in mice found that they discriminate the consonants $(/ \mathrm{g} /$ and $/ \mathrm{b} /)$ and still recognize them when combined with different vowels or emitted by other speakers. ${ }^{5}$ Nevertheless, understanding the neuronal correlates of invariant perception of complex sounds such as words, requires active recognition during neurophysiological recordings.

Studies in primates have shown that auditory processing travels from the auditory cortex to the frontal lobe via the ventral auditory stream. ${ }^{16,17}$ In humans, the ventral stream groups syllables into words during speech recognition. ${ }^{18,19}$ The prefrontal cortex projects to premotor cortices, which orchestrate behaviors. ${ }^{20}$ For instance, the macaque ventral premotor cortex, homologous to Broca's human speech production area, participates in acoustic discrimination. ${ }^{21}$ Also, as a premotor cortex, the SMA participates in voluntary movement control, ${ }^{22-24}$ working memory, and decision making. ${ }^{25-28}$ In humans, it is also involved in acoustic imagery. ${ }^{20}$ Recently, Vergara and collaborators showed that the SMA is crucial for auditory decisions ${ }^{1}$. Therefore, the SMA is a likely candidate area for linking sounds and the invariant perception thereof. ${ }^{2}$ Here we present extracellular recordings of SMA neurons in rhesus monkeys trained to

47 discriminate sounds. During the task, the monkeys reported the appearance of a target sound 
(T) presented randomly after 0 to 2 non-target sounds (nT). We evaluated the neuronal responses to various vLS of T and nT to which the monkeys had had no previous exposure (e.g. a sound uttered by different emitters). We observed that the monkeys perceived vLS as the originally learned sounds (LS), and that the SMA's neuronal responses were correlated with the animals' invariant perceptions. Our results suggest that the SMA associates incoming unexperienced sounds with the closest known category.

\section{RESULTS}

We trained two rhesus monkeys to categorize numerous sounds as $\mathrm{T}$ or $\mathrm{nT}$ during an acoustic recognition task (Fig. 1a; see Methods). The monkeys released a lever for a $\mathrm{T}$ in the first, second, or third position of a sequence (Fig. 1b). Trials with one, two, or three stimuli had an equal probability of appearing in a session $(\mathrm{p}=1 / 3)$. To assess the monkeys' capacity to identify each sound, we computed the probability of hits $\mathrm{p}$ (release $\mid \mathrm{T}$ ) and correct rejections $\mathrm{CR}=\mathrm{p}$ (no-release $\mid \mathrm{nT}$ ) (Fig. 1b, inset). Overall, each monkey performed above chance for more than 20 sounds, ${ }^{29}$ [Hits median: monkey1 $=0.97$, monkey $2=0.96 ;$ CR median: monkey 1

$64=0.98$, monkey $2=0.96$; one-sample Wilcoxon signed-rank test higher than 0.75: $\mathrm{Z}$ $($ monkey1_Hits $)=10.41, \mathrm{Z}$ (monkey1_CR $)=8.51, \mathrm{Z}$ (monkey2_Hits $)=9.63, \mathrm{Z}$ $($ monkey2_CR $)=7.87$; all p-values $<0.001]$. Overall, performance for all $\mathrm{T}$ and $\mathrm{nT}$ categories 67 ranked above $85 \%$ for both monkeys. The monkeys learned 36 sounds $(\mathrm{T}=11, \mathrm{nT}=25)$. The sounds consisted of seven artificial sounds $(\mathrm{T}=2, \mathrm{nT}=5)$, six monkey vocalizations $(\mathrm{T}=2$, $\mathrm{nT}=4)$, sixteen human words $(\mathrm{T}=6, \mathrm{nT}=10)$, and seven other animal vocalizations $(\mathrm{T}=1$, $\mathrm{nT}=6$; see reference 29 for further behavioral details). 
To test for invariant recognition of sounds, we presented the monkeys with several vLS. Figure

73

74 1c shows the T ['pwer.ta] and the nT 'grunt' spectrograms, together with three vLS. To reduce the possibility of learning the vLS, we presented each intercalated with LS no more than fifteen times per session. Ultimately, the monkeys recognized significantly above chance $84.4 \%$ of vLS ( $n=45$; Wilcoxon signed-rank test; Benjamini-Hochberg FDR corrected q-value $=0.01)$. This result means that the animals succeeded in categorizing vLS as either T or nT. However, to verify whether the monkeys perceived vLS as an invariant of LS, we sought performance differences between each of the nine LS and their corresponding five vLS (Fig. 1d). Overall, the monkeys recognized $71 \%$ vLS of LS (pairwise multiple Wilcoxon rank-sum comparison tests, Benjamini-Hochberg FDR correction, q value $=0.01$ ). It is noteworthy that the 'woof' category was highly biased towards false alarms during nT vLS since the monkeys only recognized 1 out of 5 vLS for the LS 'woof.' Overall, with the exceptions of the vLS 'woof' and the vLS ['xaw.la], the monkeys invariantly perceived most vLS as LS. (Fig. 1e) in order to determine whether the neural responses showed invariance to vLS. Figure 2a shows the raster plots of a neuron's responses to LS and vLS (left and right panels, respectively). Here, the peristimulus time histograms (PSTHs) in Fig. 2b describe similar response patterns of increased firing rates in all T regardless of whether they were LS or vLS. Interestingly, the neuron fired after the vLS 'woof' up to the activity level of the T (dashed line). Then, at the beginning of the visual cue, the firing rate fell to nT levels. Notably, neither the monkeys nor the neurons correctly classified the 'woof' sound as nT. 
responses to all $\mathrm{T}$ to almost zero spikes/second. However, it was unaffected by $\mathrm{nTs}$, including vLS of 'woof,' which means that in this case the excitatory and not the inhibitory activity was biased during the false alarms.

So that we might evaluate the extent to which the neuronal population also coded $\mathrm{T}$ and nT as invariant, i.e. regardless of their particular identities, we performed an $F$-statistic analysis. Figure 3a shows the number of neurons coding for sounds or categorical decisions during LS or vLS. Fifty-three out of the 65 recorded neurons (81.5\%) showed categorical responses to LS and 49 to vLS (75.4\%). However, 2 neurons (3\%) coded for a particular T or nT LS and $6(9 \%)$ for $\mathrm{nT}$ vLS. Figure $3 \mathrm{~b}$ presents the periods in which the neuronal population codes for $\mathrm{T}$ and $\mathrm{nT}$ in LS and vLS. Interestingly, the f-statistic values for LS categorical responses were higher than vLS, thus showing a more robust difference between $\mathrm{T}$ and $\mathrm{nT}$ rather than an effect of the number of neurons which was almost the same for LS and vLS conditions.

We also performed dPCA (see methods) in order to verify whether the neuronal population can code for acoustic identities within categorical representations. The population activity observed with dPCA showed similar patterns for LS and vLS (Figure 3c). Here, the three principal components reflect categorical coding of T. However, this analysis did not show tuning to particular T or nT sounds. One exception occurred at the third component of vLS where it was observed that the 'woof' stands out from the nT distribution. As described in Figure $2 b$, the neuronal population seems to explain the monkey's false alarms. These results suggest that invariant perception in individual and population neuronal activity does not depend on recognition of the timbre or modulations of particular emitters. Instead, invariant perception relies on a more general representation of each category. 
121 In this regard, to test whether the neuronal responses were correlated with the monkeys' behavior, we calculated Spearman correlations between behavior and the firing rates of LS and correlations throughout the task's periods and each neuron. The mean population's rho values varied similarly over time (i.e. Pearson's $\mathrm{R}=0.90, \mathrm{p}<0.001$ ). This implies that the neuronal population relates the task's events with the monkey's behavior for both LS and vLS. Moreover, the neurons that engaged across the events showed similar LS and vLS dynamics (Figure 4c; Pearson's $\mathrm{R}=0.86, \mathrm{p}<0.001$ ). Overall, these results suggest that individual and population neuronal activity correlates with invariant perception in rhesus monkeys.

\section{DISCUSSION}

133 Our results demonstrate that rhesus monkeys correctly categorized sounds to which they had 134 had no previous exposure as different variants of learned sounds. This result is significant 135 because in nonhuman primates, vocal production is highly stereotyped and probably 136 genetically determined. ${ }^{30-34}$ Numerous studies report limited acoustic recognition in nonhuman 137 primates ${ }^{35-37}$; however, emerging evidence suggests long-term plasticity in vocal learning in 138 adult monkeys exposed to vocalizations during development. ${ }^{38,39}$ For example, in a 139 match-to-sample task, macaques were better at conspecific calls than other vocalizations or 140 sounds. $^{40}$ Although we used similar sounds, we did not find performance biases towards 141 conspecific vocalizations. One possible explanation is that with the experiments of $\mathrm{Ng}$ et al., 142 discrimination of monkey vocalizations was better because those sounds had existed in 143 long-term memory since birth. In contrast, working memory alone was probably more 144 demanding and required different brain circuits. In our task, there was no working memory 145 involved. After the monkeys learned numerous sounds that may or may not be ethologically 
146 relevant, they practiced for many days until they consistently achieved performance above

$14790 \%$. Therefore, we expect different processing of short and long-term memories in

148 primates. ${ }^{41-43}$ Future studies may give insight into those differences.

Although there are no reports of perceptual invariance of sounds in non-human primates, our results show that the behavior and SMA responses to LS and vLS were invariant.

In other words, the SMA activity supports the constant perception of sounds. This result is similar to experiments in primary auditory areas demonstrating perceptual invariance during the recognition of vowels. ${ }^{4}$ However, our work does not show that the SMA represents particular sounds but rather that it directs actions based on acoustic recognition. Notably, those sounds are recognized when they relate to the motor actions triggered by known sounds. In other words, the SMA relates sounds to behaviors. ${ }^{1}$ Thus, it is reasonable to suggest that the SMA is able to sort various types of sensory information regardless of whether or not it is stored in long term circuits.

In our task, different acoustic categories lead to the release or the holding down of the lever. Therefore, each group of heterogeneous sounds produces 1 of 2 learned motor outputs. Arguably, many sounds become synonyms of "release the lever" and many others of "hold the lever." In agreement with this fact, SMA neuronal responses were correlated with two orthogonal motor plans emerging from the recognition of $\mathrm{T}$ or $\mathrm{nT}$ sounds. Noticeably, responses were invariant to $\mathrm{T}$ or $\mathrm{nT}$ groups and not to particular acoustic categories within each group. Experiments in visual areas such as the inferotemporal cortex have demonstrated that single neurons represent visual categories regardless of sensory modulations or visual perspective. ${ }^{44,45}$ Therefore, instances in the hierarchical processing of sensory information create invariant representations of images. However, in those experiments, the neurons were 
not tested during active recognition. Thus, perceptual invariance was not tested. In contrast, experiments with monkeys that actively categorized visual ${ }^{46-48}$ and auditory stimuli ${ }^{49-51}$ showed that neuronal activity in the prefrontal cortex was correlated with the animals'

174 performance. These results suggest that areas in the frontal lobe are responsible for perceptual 175 experiences while sensory areas create invariant sensory signals. Further experiments will 176 disentangle whether perceptual invariance is created at higher cortical areas fed by invariant sensory representations.

\section{CONCLUSIONS}

In rhesus monkeys, the invariant perception of sounds is more than recognition of physical identities of sounds, e.g. particular timbres and modulations. It also relies on the motor representations linked to each category. In this sense, our results suggest that the SMA is a critical instance for the auditory invariant perception of sounds such as words, because it associates un-experienced acoustic stimuli with the categorical responses triggered by known sounds.

\section{METHODS}

Ethics statement

191 All experimental protocols were performed in compliance with the Official Mexican Standard 192 Recommendations for the Care and Use of Laboratory Animals (NOM-062-ZOO-1999) and approved by the Internal Committee for the Use and Care of Laboratory Animals at the Institute of Cell Physiology, UNAM (CICUAL; LLS80-16). 


\section{Animals and experimental setup}

197 Two adult rhesus macaques (Macaca mulatta; one $13 \mathrm{~kg}$, ten year old male, and one $6 \mathrm{~kg}$, ten 198 year old female) participated in this study. The animals inhabited an enriched facility that 199 allowed physical interactions with other monkeys. The monkeys were restricted to water only 200 the night before experimental sessions. However, after the sessions, they received water $a d$ 201 libitum. Monkeys performed 1000 trials during three-hour sessions, at one session per day, 202 four to five days per week. Experiments took place in a soundproof booth in which a macaque sat on a primate chair, $60 \mathrm{~cm}$ away from a 21 " LCD color monitor $(1920 \mathrm{x} 1080$ resolution, 60 $\mathrm{Hz}$ refreshing rate). A Yamaha MSP5 speaker $(50 \mathrm{~Hz}-40 \mathrm{kHz}$ frequency range) set $15 \mathrm{~cm}$ above and behind the monitor delivered acoustic stimuli at $\sim 65 \mathrm{~dB}$ SPL measured at the monkey's ear level. Additionally, a Logitech® Z120 speaker was positioned directly below the Yamaha speaker in order to render white background noise at $\sim 55 \mathrm{~dB}$ SPL. Finally, a metal spring-lever at the monkeys' waist level captured their responses.

210 Behavioral task

211 We trained two rhesus macaques in an acoustic recognition task that consisted of categorizing sounds presented in trials of 1 to 3 sounds as target (T) or non-target (nT). At each trial, a $3^{\circ}$-aperture gray circle appeared at the monitor's center, after which the monkey pressed the

214 lever. After a variable delay of 0.5 to $1 \mathrm{~s}$, a $0.5 \mathrm{~s}$ sound was heard, followed by a $0.5 \mathrm{~s}$ delay 215 and a $0.5 \mathrm{~s}$ period when the gray circle turned green. If the sound consisted of a $\mathrm{T}$, the macaque 216 released the lever within a $0.8 \mathrm{~s}$ response window commencing with the green cue. However, 217 if the sound was an nT, the monkey kept the lever down and waited for the next sound. We 218 presented the $\mathrm{T}$ at the 1 st, 2 nd, or the 3 rd position with equal probability, i.e. $\mathrm{p}(\mathrm{T} \mid$ position $)=$ 219 1/3. Releases before a $\mathrm{T}$ created false alarms, leading to different new trials. We required that 
the monkeys perform above an $80 \%$ hit rate before electrophysiological recordings. The task's programming was in LabVIEW 2014 (64-bit SP1, National Instruments®).

222

223

Acoustic stimuli

224 The sounds were recorded in our laboratory or downloaded from free online libraries 225 (https://freesound.org/). They consisted of words, monkey vocalizations, other animal 226 vocalizations, and artificial sounds $(\mathrm{n}=37$, Supplementary Table 1$)$. The sample rate was 44.1 $\mathrm{kHz}$; cutoff frequencies, $100 \mathrm{~Hz}$ to $20 \mathrm{kHz}$, compressed or extended to $0.5 \mathrm{~s}$, and equalized to the same root-mean-square (RMS) amplitude value (Adobe Audition ${ }^{\circledR}$ version 6.0). We selected $4 \mathrm{~T}$ and $5 \mathrm{nT}$ from the pool of learned sounds in order to perform statistical repetitions (ten times each sound in a set). The phonetic nomenclature of Spanish words proceeded from the automatic phonetic transcriptionist created by Xavier López Morrás sounds, we presented them with 5 versions of each learned sound.

Neuronal recordings

We performed extracellular recordings of single SMA neurons in monkey 2. We positioned a $20 \mathrm{~mm}$ diameter recording chamber above the stereotaxic coordinates of the SMA (Paxinos G 2009) compared to monkey 2 MRI (IA $=27 \mathrm{~mm}$, left hemisphere: lateral $6 \mathrm{~mm}$ ). We used an array of 5 independently movable microelectrodes (1-3 M $\Omega$; Thomas Recording $®)$ inserted at

240 different locations in each session. We sampled extracellular membrane potentials at $40 \mathrm{kHz}$ and performed offline sorting using Plexon sorter software (Plexon®). 
244 To assess the monkeys' capacity to identify each sound, we computed the probability of hits

$245[\mathrm{p}($ release $\mid \mathrm{T}), \mathrm{T}]$ and Correct Rejections $[\mathrm{CR}=\mathrm{p}($ no-release $\mid \mathrm{nT}), \mathrm{nT}]$, we performed a

246 one-sample Wilcoxon signed-rank test. To verify the difference between the LS and vLS, we

247 calculated the number of vLS that were different from LS (pairwise multiple Wilcoxon rank-

248 sum comparison tests, Benjamini-Hochberg FDR correction, q value $=0.01$ ). categories or acoustic identities, we calculated $F$-statistics in a one-way ANOVA of the T vs. $\mathrm{nT}$ and each sound. We calculated the mean firing rate in 200-ms windows in steps of $20 \mathrm{~ms}$. In order to avoid biases in the $F$-statistic due to the number of trials, we only included the same number in all classes. We repeated the analysis 1000 times to create an $F$-statistic distribution. We compared the actual distribution against an $F$-statistic random distribution obtained by shuffling the stimulus labels 1000 times to determine the significant $F$-statistic bins. A neuron was significant if confidence intervals (95\%) did not overlap in at least one time-bin. effects of the task components and of acoustic identities on the population responses. During the supervised stage, dPCA decomposes the neural activity for each variable in covariance matrices of different marginalizations. Then, for each marginalization matrix, unsupervised analysis is similar to a PCA. We marginalized the population activity for T, nT, and time, from the beginning of the sounds to the monkeys' responses, in $200 \mathrm{~ms}$ windows every $20 \mathrm{~ms}$. dPCA differentiates the matrices from the decoder (D) and the encoder $(F)$ for each parameter by minimizing the loss function:

$$
L_{\phi}=\left\|\underline{X}_{\phi}-F_{\phi} D_{\phi} \underline{X}\right\|
$$


268 Where $\phi$ denotes the marginalization for each parameter and $X$, the mean-centered population matrix. Each component represents an amount of firing rate variance. The axes obtained by the decoder and the encoder allow data reduction in a few features, capturing the most significant variance of each parameter. sets. The testing sets consisted of random inclusions of 1 trial from each category ( $\mathrm{T}$ and $\mathrm{nT}$ ). The training set was the mean of the remaining tests of each class. We performed dPCA to the training set in order to obtain the decoding axes. Then, we projected the testing set on these axes and classified them according to the closest category mean (T or nT). We repeated this procedure 1000 times and measured the proportion of correct classification. We shuffled 100 times for significance.

To test whether the neuronal responses were correlated with behavior, we computed Spearman correlations between the monkeys' performance and neuronal firing rates of LS and vLS, in $200 \mathrm{~ms}$ windows, every 20 ms. Finally, we calculated a Pearson correlation between the resulting mean Spearman's rho of LS and vLS in order to assess for similarities.

\section{ACKNOWLEDGMENTS}

288 We are grateful for the financial support provided by CONACYT CB-256767, and Programa de Apoyo a Proyectos de Investigación e Innovación Tecnológica [Support Program for 
Programa de Doctorado en Ciencias Biomédicas [doctoral program in biomedical sciences], at

Universidad Nacional Autónoma de México (UNAM) and he was supported by CONACYT 229866. The data in this work are part of his doctoral dissertation.

296

\section{AUTHOR CONTRIBUTIONS}

298

299

JM, IM, TF, and LL performed experiments, JM, IM, JV, and JP analyzed data, LL designed the paradigm, TF programmed the task, while JM and LL wrote the paper.

301

\section{CONFLICT OF INTEREST STATEMENT}

303

304

The authors declare no competing financial interests.

305

306

\section{BIBLIOGRAPHY}

307

308 1. Vergara, J., Rivera, N., Rossi-Pool, R. \& Romo, R. A Neural Parametric Code for 309 Storing Information of More than One Sensory Modality in Working Memory. Neuron 89, 54-62 (2016).

2. Feng, G., Gan, Z., Wang, S., Wong, P. C. M. \& Chandrasekaran, B. Task-General and Acoustic-Invariant Neural Representation of Speech Categories in the Human Brain. Cereb. Cortex 28, 3241-3254 (2018).

3. Elie, J. E. \& Theunissen, F. E. Meaning in the avian auditory cortex: Neural representation of communication calls. Eur. J. Neurosci. 41, 546-567 (2015).

316 4. Town, S. M., Wood, K. C. \& Bizley, J. K. Sound identity is represented robustly in auditory cortex during perceptual constancy. Nat. Commun. 9, (2018). 
318 5. Saunders, J. L. \& Wehr, M. Mice can learn phonetic categories. J. Acoust. Soc. Am. 145, 1168-1177 (2019).

6. Hauser, M. D. Functional referents and acoustic similarity: Field playback experiments with rhesus monkeys. Anim. Behav. 55, 1647-1658 (1998).

7. Slocombe, K. E. \& Zuberbühler, K. Food-associated calls in chimpanzees: Responses to food types or relative food value? Anim. Behav. 72, 989-999 (2006).

8. Daniel Meliza, C. \& Margoliash, D. Emergence of selectivity and tolerance in the avian auditory cortex. J. Neurosci. 32, 15158-15168 (2012).

9. Elie, J. E. \& Theunissen, F. E. Meaning in the avian auditory cortex: Neural representation of communication calls. Eur. J. Neurosci. 41, 546-567 (2015).

10. Jiang, X., Chevillet, M. A., Rauschecker, J. P. \& Riesenhuber, M. Training Humans to Categorize Monkey Calls: Auditory Feature- and Category-Selective Neural Tuning Changes. Neuron 98, 405-416.e4 (2018).

11. Belin, P., Bodin, C. \& Aglieri, V. A "voice patch" system in the primate brain for processing vocal information? Hear. Res. 366, 65-74 (2018).

12. Belin, P., Zatorre, R. \& Pike, B. G. Voice-selectiveareas in humanauditorycortex Modulating top-down and bottom-up contributions to auditory stream segregation and integration with polyphonic music View project Neural Mechanisms of Voice Processing in Marmosets. View project. https://www.researchgate.net/publication/235653597 (2000).

13. Leaver, A. M. \& Rauschecker, J. P. Cortical representation of natural complex sounds: Effects of acoustic features and auditory object category. J. Neurosci. 30, 7604-7612 (2010).

341 14. Ortiz-Rios, M. et al. Functional MRI of the vocalization-processing network in the macaque brain. Front. Neurosci. 9, (2015). 
15. Petkov, C. I. et al. A voice region in the monkey brain. Nat. Neurosci. 11, 367-374 (2008).

16. Bizley, J. K. \& Cohen, Y. E. The what, where and how of auditory-object perception. Nat. Rev. Neurosci. 14, 693-707 (2013).

17. Romanski, L. M., Bates, J. F. \& Goldman-Rakic, P. S. Auditory belt and parabelt projections to the prefrontal cortex in the rhesus monkey. J. Comp. Neurol. 403, 141157 (1999).

18. Leonard, M. K. \& Chang, E. F. Dynamic speech representations in the human temporal lobe. Trends Cogn. Sci. 18, 472-479 (2014).

19. Repp, B. H. Categorical Perception: Issues, Methods, Findings. in vol. 10 243-335 (Elsevier, 1984).

20. Lima, C. F., Krishnan, S. \& Scott, S. K. Roles of Supplementary Motor Areas in Auditory Processing and Auditory Imagery. Trends Neurosci. 39, 527-542 (2016).

21. Lemus, L., Hernández, A. \& Romo, R. Neural encoding of auditory discrimination in ventral premotor cortex. Proc. Natl. Acad. Sci. U. S. A. 106, 14640-14645 (2009).

22. Lara, A. H., Cunningham, J. P. \& Churchland, M. M. Different population dynamics in the supplementary motor area and motor cortex during reaching. Nat. Commun. 9, (2018).

23. Nachev, P., Kennard, C. \& Husain, M. Functional role of the supplementary and presupplementary motor areas. Nat. Rev. Neurosci. 9, 856-869 (2008).

24. Shima, K. \& Tanji, J. Neuronal activity in the supplementary and presupplementary motor areas for temporal organization of multiple movements. J. Neurophysiol. 84, 2148-2160 (2000).

25. Morán, I., Perez-Orive, J., Melchor, J., Figueroa, T. \& Lemus, L. Auditory Decisions in 
doi:10.1101/2020.10.20.347864.

26. Hernández, A., Zainos, A. \& Romo, R. Temporal evolution of a decision-making process in medial premotor cortex. Neuron 33, 959-972 (2002).

27. Lemus, L. et al. Neural correlates of a postponed decision report. Proc. Natl. Acad. Sci. U. S. A. 104, 17174-17179 (2007).

28. Romo, R., Merchant, H., Zainos, A. \& Hernández, A. Categorical perception of somesthetic stimuli: Psychophysical measurements correlated with neuronal events in primate medial premotor cortex. Cereb. Cortex 7, 317-326 (1997).

29. Melchor, J., Morán, I., Figueroa, T. \& Lemus, L. Perceptual Invariance of Words and

Other Learned Sounds in Non-human Primates. bioRxiv 805218 (2019) doi:10.1101/805218.

30. Brockelman, W. Y. \& Schilling, D. Inheritance of stereotyped gibbon calls. Nature 312, 634-636 (1984).

31. Hammerschmidt, K. \& Fischer, J. Baboon vocal repertoires and the evolution of primate vocal diversity. J. Hum. Evol. 126, 1-13 (2019).

32. Nieder, A. \& Mooney, R. The neurobiology of innate, volitional and learned vocalizations in mammals and birds. Philosophical Transactions of the Royal Society B: Biological Sciences vol. 375 (2020).

33. Owren, M. J., Dieter, J. A., Seyfarth, R. M. \& Cheney, D. L. 'Food' Calls Produced by Adult Female Rhesus (Macaca Mulatta) and Japanese (M. Fuscata) Macaques, their Normally-Raised Offspring, and Offspring Cross-Fostered Between Species. Behaviour 120, 218-231 (1992).

34. Zador, A. M. A critique of pure learning and what artificial neural networks can learn from animal brains. Nat. Commun. 10, 3770 (2019).

392 35. Fritz, J., Mishkin, M. \& Saunders, R. C. In search of an auditory engram. Proc. Natl. 
Acad. Sci. 102, 9359-9364 (2005).

36. Scott, B. H., Mishkin, M. \& Yin, P. Monkeys have a limited form of short-term memory in audition. Proc. Natl. Acad. Sci. U. S. A. 109, 12237-12241 (2012).

37. Wright, A. A. Auditory list memory and interference processes in monkeys. J. Exp. Psychol. Anim. Behav. Process. 25, 284-296 (1999).

38. Takahashi, D. Y., Liao, D. A. \& Ghazanfar, A. A. Vocal Learning via Social Reinforcement by Infant Marmoset Monkeys. Curr. Biol. 27, 1844-1852.e6 (2017).

39. Zhao, L., Rad, B. B. \& Wang, X. Long-lasting vocal plasticity in adult marmoset monkeys. Proc. R. Soc. B Biol. Sci. 286, (2019).

40. Ng, C. W., Plakke, B. \& Poremba, A. Primate auditory recognition memory performance varies with sound type. Hear. Res. 256, 64-74 (2009).

404

41. Fritz, J. B., Malloy, M., Mishkin, M. \& Saunders, R. C. Monkey's short-term auditory memory nearly abolished by combined removal of the rostral superior temporal gyrus and rhinal cortices. Brain Research vol. 1640 289-298 (2016).

42. Muñoz-López, M., Insausti, R., Mohedano-Moriano, A., Mishkin, M. \& Saunders, R. C. Anatomical pathways for auditory memory II: Information from rostral superior temporal gyrus to dorsolateral temporal pole and medial temporal cortex. Front. Neurosci. 9, 1-21 (2015).

43. Munoz-Lopez, M. M., Mohedano-Moriano, A. \& Insausti, R. Anatomical pathways for auditory memory in primates. Frontiers in Neuroanatomy (2010) doi:10.3389/fnana.2010.00129.

44. Freiwald, W. A. \& Tsao, D. Y. Functional compartmentalization and viewpoint generalization within the macaque face-processing system. Science (80-. ). 330, 845$851(2010)$.

417 45. Hesse, J. K. \& Tsao, D. Y. The macaque face patch system: a turtle's underbelly for the 
brain. Nat. Rev. Neurosci. 21, 695-716 (2020).

46. Seger, C. A. \& Miller, E. K. Category Learning in the Brain. Annu. Rev. Neurosci. 33, 203-219 (2010).

421

422

423

424

425

426

427

47. Cromer, J. A., Roy, J. E. \& Miller, E. K. Representation of Multiple, Independent Categories in the Primate Prefrontal Cortex. Neuron 66, 796-807 (2010).

48. Roy, J. E., Buschman, T. J. \& Miller, E. K. PFC neurons reflect categorical decisions about ambiguous stimuli. J. Cogn. Neurosci. 26, 1283-1291 (2014).

49. Russ, B. E., Orr, L. E. \& Cohen, Y. E. Prefrontal Neurons Predict Choices during an Auditory Same-Different Task. Curr. Biol. 18, 1483-1488 (2008).

50. Lee, J. H., Russ, B. E., Orr, L. E. \& Cohen, Y. E. Prefrontal activity predicts monkeys' decisions during an auditory category task. Front. Integr. Neurosci. 3, 1-12 (2009).

51. Huang, Y. \& Brosch, M. Associations between sounds and actions in primate prefrontal cortex. Brain Res. 1738, 1-22 (2020).

52. Kobak, D. et al. Demixed principal component analysis of neural population data. Elife (2016) doi:10.7554/eLife.10989.

\section{FIGURE LEGENDS}

Figure 1. Auditory recognition task and behavioral performance. a) Events in a trial. First, a visual cue (grey circle) appears at the center of the screen, indicating that the monkey should press and hold down the lever. After a variable period (0.5 to $1 \mathrm{~s})$, a playback of 1-3 sounds commenced. Each sound continued with a $0.5 \mathrm{~s}$ delay and a $0.5 \mathrm{~s}$ green cue that replaced the grey circle. The monkey received a reward for releasing the lever within $0.7 \mathrm{~s}$ after the beginning of the green cue. Color code: orange (T), purple (nT), green (go-cue). b) Types of trials. The T could appear in the first, second, or third position during a trial. Inset: Behavior 
as a function of the lever release in response to $\mathrm{T}$ and $\mathrm{nT}$. FA, false alarms; CR, correct

444 rejections. c) Spectrograms of T and nT sounds and some acoustic versions (upper and lower rows, respectively). d) Mean hit rates for learned T and CR for learned nT (orange and purple bars, respectively) and vLS (grey bars). The central horizontal line demarcates acoustic

447 recognition by chance at 50\%. Black asterisks: performance different from LS. Red asterisk:

448 an exceptional sound that was recognized as a T. e) Structural MRI of monkey 1; SMA 449 indicated.

Figure 2. Responses of SMA neurons during the recognition of learned and version

sounds. a) Raster plot of a neuron's responses during trials of LS and vLS (left and right panels, respectively). Each line is a trial aligned to the beginning of sounds. Each dot is an action potential. The sounds were presented randomly during the experiment but shown here in blocks of $\mathrm{T}$ and nT sounds (upper and lower groups, respectively. b) PSTHs of the neuron in $\mathbf{a}$. CR, correct rejections, and FA, false alarms of vLS 'woof.' c) PSTHs of a second neuron recorded

457 in the same session.

Figure 3. Categorical responses of the SMA population. a) Neurons with categorical SMA mean $F$-statistic values as a function of time. Black lines: categorical responses. Orange:

462 T identities. Blue: nT identities. Continuous lines: LS. Dashed lines: vLS. c) Each row: first 463 three demixed principal components of the SMA neuronal population during LS and vLS. Dashed line: ‘woof’ sound. Orange colors: T. Purple colors: nT.

a) Responses of 1 neuron to LS and vLS. Insets: a time window indicated by the dashed box, 
468 where the probability of the monkey releasing the lever after each $\mathrm{T}$ and $\mathrm{nT}$ (black line) is 469 similar to neuronal responses to LS and vLS (blue line). Rho: the Spearman's correlation and 470 p significance for that temporal window. b) Significant Spearman's rho along with the task for 471 each neuron during LS and vLS. c) Times where the neuronal population recorded during LS 472 and vLS were correlated with the monkey's performance. 


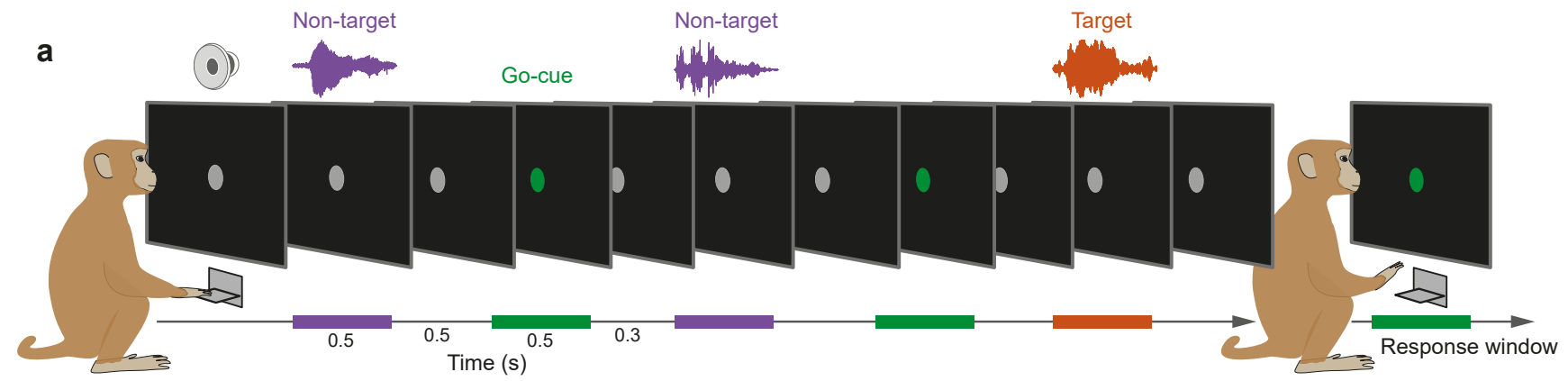

b

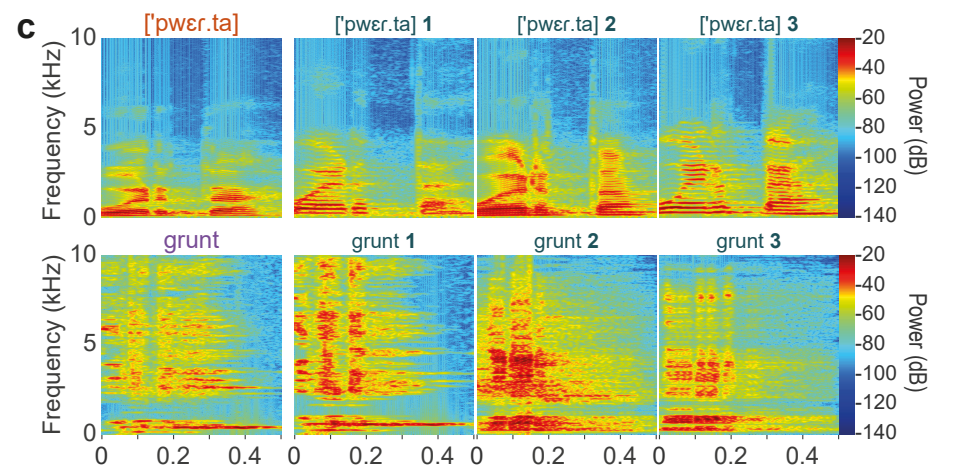

d

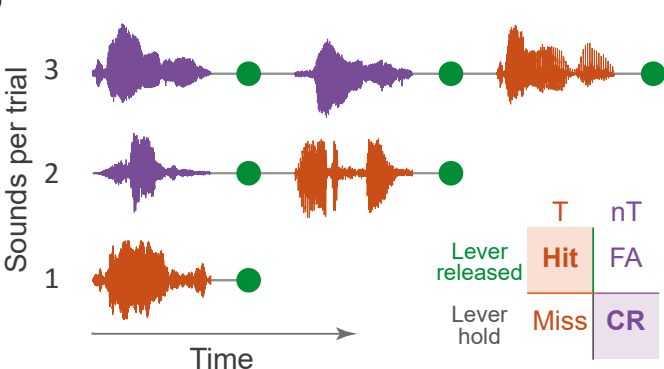

DLearned
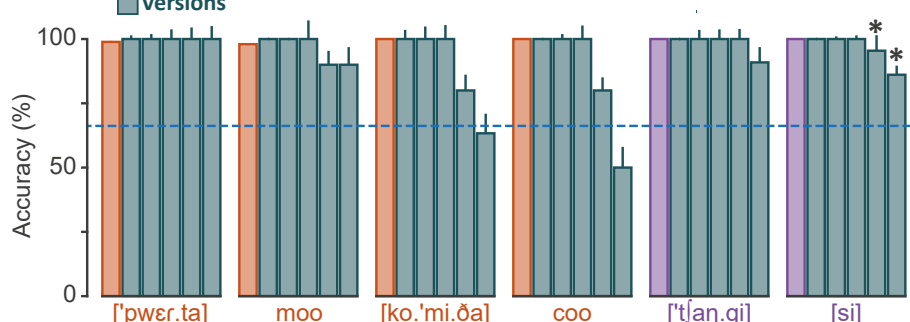

[si]
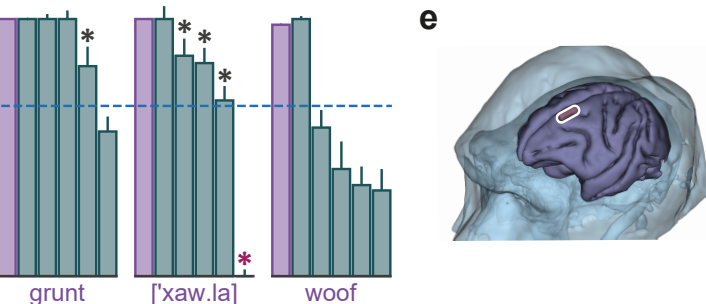

Figure 1 
bioRxiv preprint doi: https://doi.org/10.1101/2020.12.22 424045; this version posted December 22, 2020. The copyright holder for this preprint (which was not certified by peer review) is the author/funder. All rights reserved. No reuse allowed without permission.
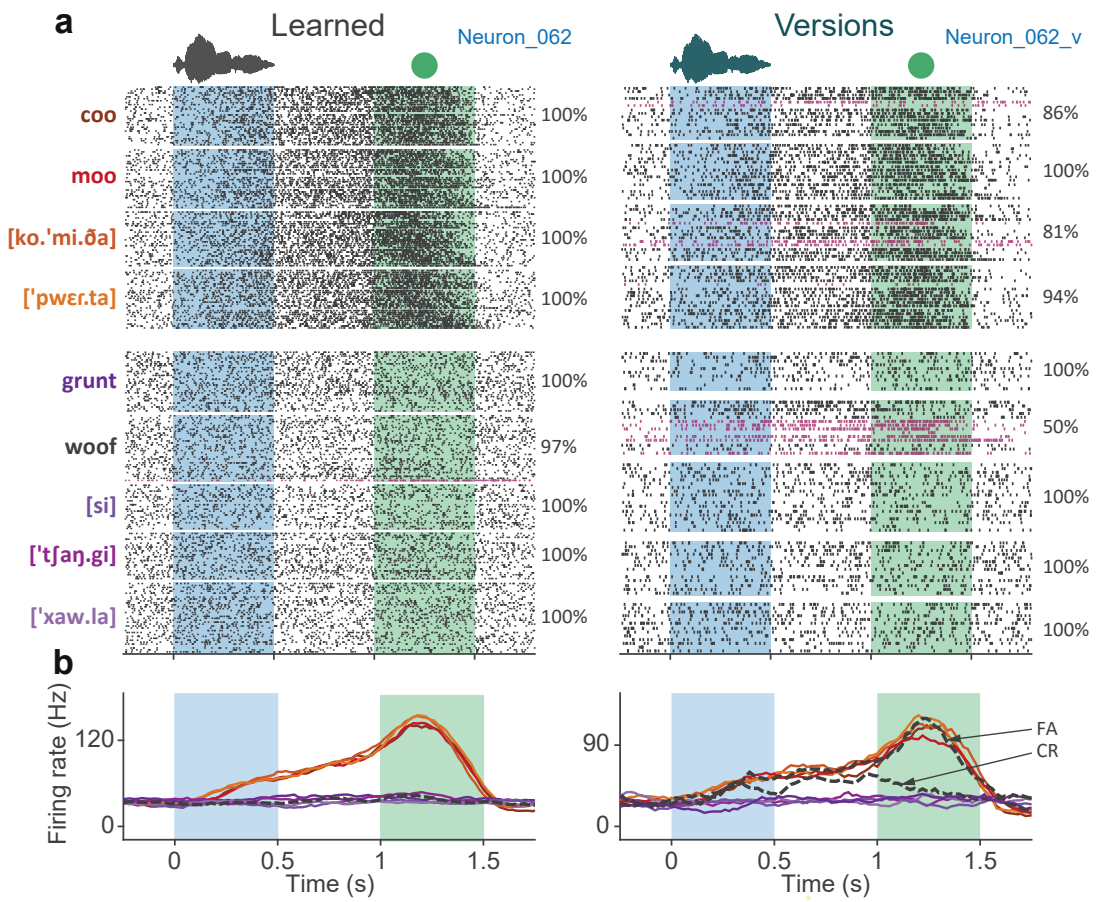

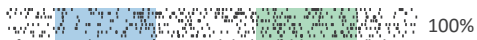

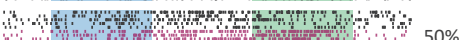

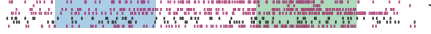

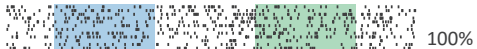
7 and

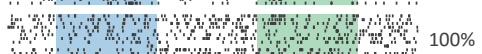
A

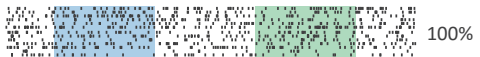
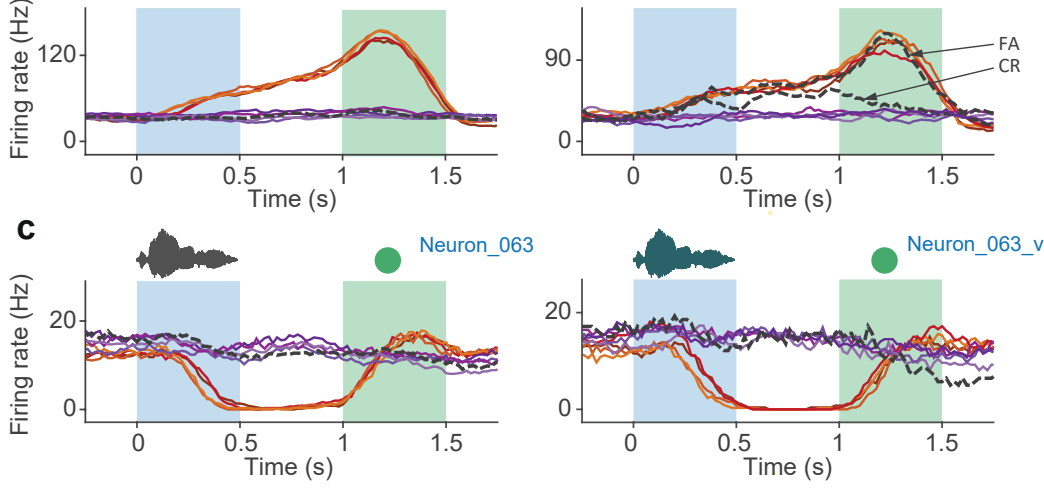

Figure 2 
a
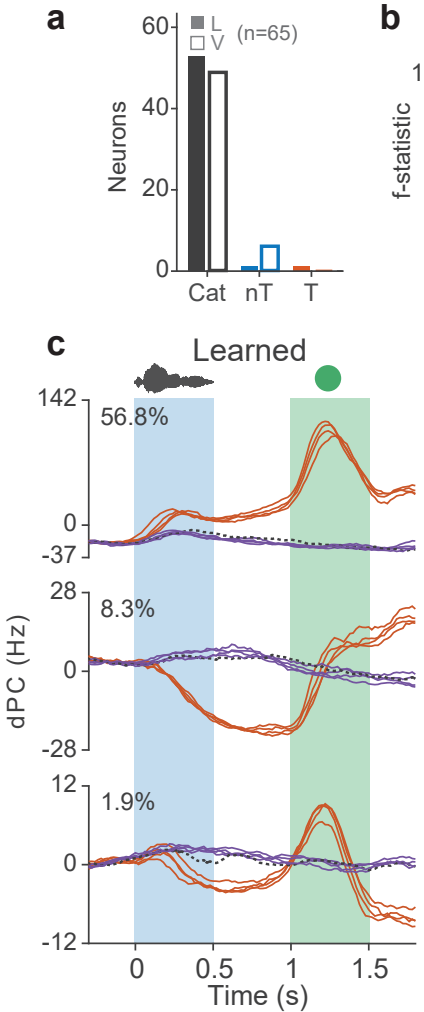

b
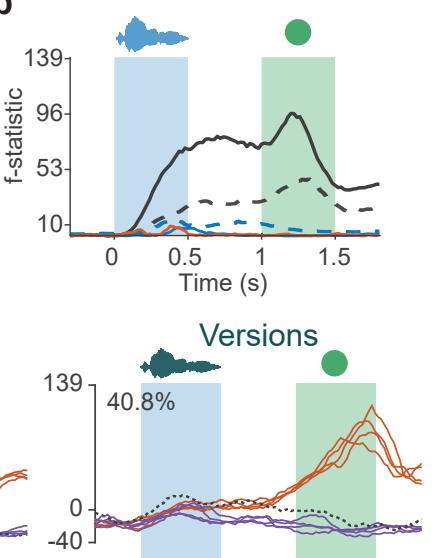

$\left.{ }^{39}\right] 10.5 \%$

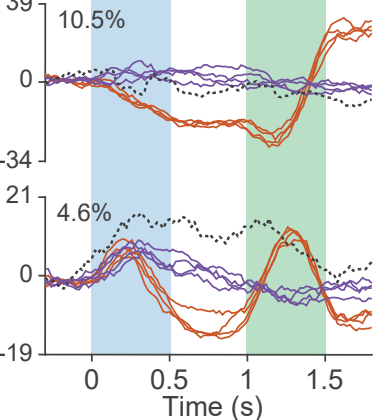

Figure 3 
bioRxiv preprint doi: https://doi.org/10.1101/2020.12.22.424045; this version posted December 22, 2020. The copyright holder for this preprint (which was not certified by peer review) is the author/funder. All rights reserved. No reuse allowed without permission.
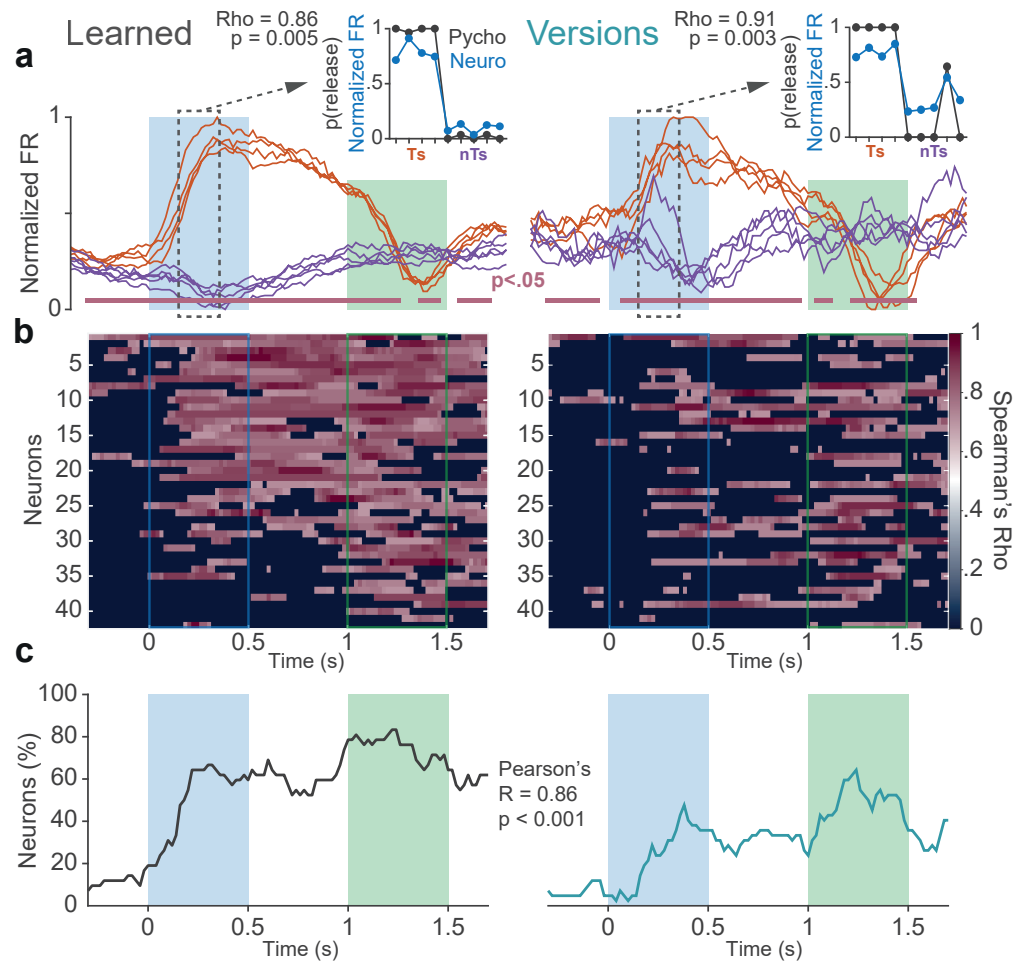

Figure 4 